\title{
Incidence and risk factors for respiratory tract bacterial colonization and infection in lung transplant recipients
}

\author{
L. Paglicci ${ }^{1} \cdot$ V. Borgo ${ }^{1} \cdot$ N. Lanzarone ${ }^{2} \cdot$ M. Fabbiani ${ }^{1} \cdot$ C. Cassol ${ }^{1,3} \cdot$ MG. Cusi $^{3,4} \cdot$ M. Valassina $^{4} \cdot$ S. Scolletta ${ }^{5}$. \\ E. Bargagli ${ }^{2}$ - L. Marchetti ${ }^{6}$ P. Paladini ${ }^{7}$ - L. Luzzi ${ }^{7}$ - A. Fossi $^{2}$ • D. Bennett ${ }^{2} \cdot$ F. Montagnani $^{1,3}$ (I)
}

Received: 1 July 2020 / Accepted: 6 January 2021 / Published online: 21 January 2021

(C) The Author(s) 2021

\begin{abstract}
To evaluate incidence of and risk factors for respiratory bacterial colonization and infections within 30 days from lung transplantation (LT). We retrospectively analyzed microbiological and clinical data from 94 patients transplanted for indications other than cystic fibrosis, focusing on the occurrence of bacterial respiratory colonization or infection during 1 month of follow-up after LT. Thirty-three percent of patients developed lower respiratory bacterial colonization. Bilateral LT and chronic heart diseases were independently associated to a higher risk of overall bacterial colonization. Peptic diseases conferred a higher risk of multidrug resistant (MDR) colonization, while longer duration of aerosol prophylaxis was associated with a lower risk. Overall, 35\% of lung recipients developed bacterial pneumonia. COPD (when compared to idiopathic pulmonary fibrosis, IPF) and higher BMI were associated to a lower risk of bacterial infection. A higher risk of MDR infection was observed in IPF and in patients with pre-transplant colonization and infections. The risk of post-LT respiratory infections could be stratified by considering several factors (indication for LT, type of LT, presence of certain comorbidities, and microbiologic assessment before LT). A wider use of early nebulized therapies could be useful to prevent MDR colonization, thus potentially lowering infectious risk.
\end{abstract}

Keywords Lung transplantation $\cdot$ Bacterial infections $\cdot$ Host bacteria interactions $\cdot$ Pulmonary fibrosis

\section{Introduction}

Lung transplant (LT) is considered a reasonable treatment option for selected patients with chronic respiratory endstage diseases. The most frequent indications for adult LT are chronic obstructive pulmonary diseases (COPD) with or without $\alpha 1$-antitrypsin deficiency, interstitial lung diseases, cystic fibrosis, and idiopathic pulmonary arterial hypertension $[1,2]$. Each one of these diseases has its own peculiar features

F. Montagnani

francesca.montagnani@unisi.it

1 Department of Medical Sciences, Infectious and Tropical Diseases Unit, Siena University Hospital, Siena, Italy

2 Department of Medical and Surgical Sciences \& Neurosciences, Respiratory Diseases and Lung Transplantation Unit, Siena University Hospital, Siena, Italy

3 Department of Medical Biotechnologies, University of Siena, Siena, Italy in term of transplant list waiting, mortality, and post-transplant survival $[3,4]$.

Despite post-LT survival rate has increased over the last years, risk of early and late post-surgical complications remains high for transplanted patients [2,5]. In particular, lung transplant recipients (LTRs) are at higher risk to develop infectious complications compared to other solid organ transplants; this can be due not only to immunosuppressive regimens and changes in anatomical structures after surgery, but

4 Department of Innovation, Experimentation and Clinical Research, Microbiology and Virology Unit, Siena University Hospital, Siena, Italy

5 Department of Emergency and Urgency, Medicine, Surgery and Neurosciences, Unit of Intensive Care Medicine, Siena University Hospital, Siena, Italy

6 Cardio-Thoracic-Vascular Department, Anesthesia and Cardio-Thoracic-Vascular Intensive Care Unit, Siena University Hospital, Siena, Italy

7 Cardio-Thoracic-Vascular Department, Thoracic Surgery Unit, Siena University Hospital, Siena, Italy 
also to constant exposure of airways to the outside environment $[6,7]$. Infectious complications represent the most frequent cause of death after graft failure within the first 30 days from transplant, and the first cause within the first year [2].

Generally, the most common infections occur following a specific pattern based on the time from $\mathrm{LT}$, as a consequence of the exposure to different risk factors over the time such as hospitalization, immunosuppression, reactivation of latent infection, and community-acquired pathogens exposure. During the early post-transplant period, infections can be related to post-surgical complications, donor or recipient's pre-existing colonizations/infections, or nosocomial-acquired organisms [6-9]. Bacterial pneumonia is the most common infection in the first 30 days from LT, related to healthcare-associated or nosocomial bacteria. This risk decreases after 6 months from LT $[7,10]$ but the possibility to be colonized and infected by a multi-drug resistant (MDR) strain increases with the duration of pre-transplant hospitalizations and post-surgical intubation $[1,8]$.

Infections are also a major cause of late mortality, and a linkage (especially for bacterial aetiologies) with chronic rejection development is hypothesized, although their pathogenic role is not fully understood $[1,11]$.

Data on etiologic agents of pneumonia after lung transplant are limited for non-cystic fibrosis patients. Most of the bacteria isolated in post-transplant infections are Gram negative (e.g., Pseudomonas aeruginosa, Klebsiella pneumoniae) with a lower incidence of Gram positive bacteria, mainly represented by methicillin resistant Staphylococcus aureus (MRSA) [8, 12].

Prevalence and type of colonization, occurrence and type of infections and prevalence of MDR bacteria in the first month after transplantation can vary between patients in relation to the type of chronic illness causing end-stage organ disease, as well as to the clinical and microbiological history before and after transplantation $[8,13]$.

The aim of the study was to evaluate the incidence of and risk factors for respiratory bacterial colonization and infections within 30 days from LT in patients not affected by cystic fibrosis.

\section{Material and methods}

Patients We retrospectively analyzed patients who underwent lung transplantation from January 1, 2001 to December 31, 2018 at the Lung Transplant Centre of the University Hospital of Siena, Tuscany, Central Italy. We included in the analysis only those subjects who had at least 18 years of age, had an available 1-month post-transplant follow-up, and complete clinical and microbiological data after transplantation. We excluded patients who underwent LT for cystic fibrosis since this disease has its own clinical features in terms of either recipient typology or pre-transplant colonization and infection etiologies, and therefore it is less comparable to the other lung transplant indications [14]. Patients were followed from the time of LT to a 1 month of follow-up and evaluated for the occurrence of bacterial respiratory colonization or infection, as below defined.

Pre-transplant data For each patient, demographic and clinical data were collected: gender, age, ethnicity, body mass index (BMI), indication for LT, type of LT (single/double), comorbidities (obesity, diabetes, arterial hypertension, dyslipidaemia, osteoporosis/osteopenia, peptic disease, cardiopathy, endocrinopathy, non-viral hepatopathy, psychiatric syndrome, nephropathy, rheumatic disease peripheral vasculopathy). We also evaluated previous hospitalizations occurring within 30 and 90 days before LT, administration of any antibiotic therapy in the pre-transplant pe$\operatorname{riod}(\leq 7,30,90$ days) and the use of respiratory devices (orotracheal intubation, tracheostomy) and/or extracorporeal membrane oxygenation (ECMO) during the pre-transplant phase or during the surgery.

When retrospectively available, microbiological data from donors were also collected.

Post-transplant data The following post-transplant data were collected: presence of any devices (naso-gastric tube, NGT; central venous catheter, CVC; vesical catheter, VS; continuous veno-venous hemofiltration, $\mathrm{CVVH}$; tracheostomy; orotracheal intubation; pleural drainage), length of hospital stay (LOS), occurrence of acute rejection, occurrence of primary graft dysfunction (PGD), and its grade (1-2-3) [15].

Microbiological data For each patient, we analyzed all respiratory samples [sputum, bronchoalveolar lavage (BAL) or bronchial aspirate (BAS)] that were collected for bacterial culture during the first month of follow-up post-LT. Bronchoscopy was regularly performed at day 1 after LT and every $24-48 \mathrm{~h}$ thereafter depending on clinical judgment, in case of symptoms or signs suggestive of lung infection and/ or on the need to remove bronchial secretions. Bronchoalveolar lavage was always performed in association to transbronchial lung biopsy: all patients underwent surveillance biopsies at day 25-35 and thereafter on clinical demand. Airway samples, if collected during bronchoscopy, were routinely cultured.

Respiratory bacterial colonization was defined as isolation of bacteria from respiratory samples in the absence of significant laboratory and/or clinical alterations supporting diagnosis of infection. Respiratory bacterial infection was defined as isolation of bacteria from respiratory samples in the presence of significant laboratory and/or clinical alterations consistent with infection (e.g., fever, worsening cough and sputum 
production, dyspnoea, leucocytosis, increased $\mathrm{C}$ reactive protein, and/or procalcitonin).

For each bacterial isolate, we analyzed antimicrobial resistance profile, available from medical reports. The definition of multi-drug resistant (MDR), extensively drug resistant (XDR), pan-drug resistant (PDR) strain was based on the following criteria [16]: MDR was defined as acquired nonsusceptibility to at least one agent in three or more antimicrobial categories, XDR was defined as non-susceptibility to at least one agent in all but two or fewer antimicrobial categories (i.e., bacterial isolates remain susceptible to only one or two categories) and PDR was defined as non-susceptibility to all agents in all antimicrobial categories.

Prophylactic strategies During the study period 2001 to 2018, our internal protocol (hereinafter, standard prophylaxis) consisted of universal prophylaxis with an extended spectrum cephalosporin, usually ceftazidime, plus a glycopeptide (vancomycin as first line therapy) started $1 \mathrm{~h}$ before the surgery and maintained for some days after surgery, depending on clinical judgment. Furthermore, prophylaxis with cotrimoxazole was usually started after 1 week to avoid the risk of Pneumocystis jirovecii pneumonia, in association with acyclovir universal prophylaxis. Finally, CMV prophylaxis was administered in case donor/ recipient mismatch (D+/R-); in all other cases, a preemptive approach was adopted. Tailored approaches were used in case of donor or recipient's previous bacterial isolates, based on the antimicrobial resistance profile. Use of voriconazole varied over the study years and on the basis of individual characteristics of patients, between universal prophylaxis and pre-emptive approach.

In case of previous isolates reporting susceptibility to polymixins (colistin), aerosol prophylaxis with this drug was prescribed in addition to systemic prophylaxis in selected patients. Furthermore, accordingly to our protocol, most patients received antimicrobial prophylaxis with nebulized taurolidine in the early post-transplant. This kind of strategy has been developed from the literature data available for patients transplanted for cystic fibrosis [17-20].

Statistical analysis Descriptive statistics [number, proportion, median, interquartile range (IQR)] were used to describe the baseline characteristics of patients. Categorical variables were compared by chi-square $\left(\chi^{2}\right)$, Fisher exact test, and McNemar test when necessary. For the comparison of continuous variables, the Mann-Whitney $U$ test was used. Factors associated to bacterial respiratory colonization and infection were analyzed by logistic regression. Only $p$ values $<0.05$ were considered to be statistically significant. All analyses were performed using the SPSS version 18.0 software package (SPSS Inc., Chicago, IL, USA).

\section{Results}

\section{Population characteristics at baseline}

Overall, 161 patients underwent LT during the study period. Thirty-one (19.5\%) subjects were excluded since they were transplanted for cystic fibrosis, $36(22.3 \%)$ were excluded for missing or incomplete clinical and/or microbiological data. Finally, a total of 94 patients fulfilled all the inclusion criteria and were included in the study. Table 1 summarizes their baseline characteristics. The studied population was mainly constituted by male $(n=59 ; 68.2 \%)$ patients with a median age of 56.5 years (IQR 49.8-61) and a median pre-transplant BMI of $24 \mathrm{~kg} / \mathrm{m}^{2}$ (IQR 20-29). All the patients were Caucasian. Overall, $53.2 \%(n=50)$ of recipients had an optimal BMI $\left(18.5-24.99 \mathrm{~kg} / \mathrm{m}^{2}\right)$ in pre-transplant period, $41.5 \%$ were overweighed (BMI $25-29.99 \mathrm{~kg} / \mathrm{m}^{2}$ ), or mildly obese (BMI 30-34.99 kg/m²) (respectively, $n=19$ and $n=20$ ). A percentage of $5.3(n=5)$ of patients were classified as underweighted during the pre-transplant evaluation and no patient had a baseline BMI greater than $33 \mathrm{~kg} / \mathrm{m}^{2}$.

The most common indication for LT was idiopathic pulmonary fibrosis (IPF) $(n=41 ; 43.6 \%)$, followed by COPD $(n=24 ; 25.5 \%)$. Twenty-nine patients $(30.8 \%)$ underwent LT for other indications, notably interstitial lung diseases different from IPF in 55\% of the remaining cases $(n=16 / 29)$ (e.g., non-specific interstitial pneumonia, NSIP; allergic extrinsic alveolitis; interstitial diseases in patients with systemic sclerosis and autoimmune arthritis; graft versus host disease), sarcoidosis $(n=3 / 29,10.3 \%)$, pulmonary microlitiasis $(n=3 /$ $29,10.3 \%)$, pulmonary Langerhans cell histiocytosis $(n=2 /$ $29,6.9 \%)$, non-CF bronchiectasis $(n=2 / 29,6.9 \%)$, lymphangioleiomyomatosis $(n=1 / 29,3.4 \%)$, primary ciliary dyskinesia $(n=1 / 29,3.4 \%)$, obliterans bronchiolitis $(n=1 / 29$, $3.4 \%)$.

On the total, 78 patients (83\%) had at least one comorbidity. The most common was osteoporosis/osteopenia $(n=48$; $51.1 \%)$, followed by diabetes $(n=23,24.5 \%)$, obesity $(n=20$, $21.3 \%)$, arterial hypertension $(n=20,21.3 \%) ; 11.7 \%$ of patients had peptic diseases (gastroesophageal reflux, gastritis or peptic ulcer). Most of patients underwent single lung transplantation $(55.3 \%, n=52 / 94)$.

During the pre-transplant screening evaluation, $13.8 \%$ of recipients $(n=13)$ had a bacterial respiratory colonization, with a small prevalence of MDR strains ( $n=2 / 90$ available reports, $2.2 \%$ ). Respiratory infections were found in $6.6 \%$ of cases $(n=6)$, with one case of infection by MDR bacteria. Available retrospective microbiological data from donors showed $27.4 \%(n=23 / 84)$ cases of colonization and $7.4 \%$ $(n=6 / 81)$ cases of infection. On the basis of accessible microbiological results, only $2.5 \%(n=2 / 80)$ of isolates from colonized donors were MDR bacteria; no MDR bacteria were revealed from infected donors. 
Table 1 Baseline characteristics of the study population $(n=94)$

\begin{tabular}{|c|c|}
\hline Characteristics & $N(\%)$ or median (IQR) \\
\hline Age, years & $56.5(49.8-61)$ \\
\hline Male: & $59(62.8)$ \\
\hline Pre-transplant BMI, $\mathrm{kg} / \mathrm{m}^{2}$ & $24(20-29)$ \\
\hline \multicolumn{2}{|l|}{ Indication for transplant: } \\
\hline - IPF & $41(43.6)$ \\
\hline - COPD & $24(25.5)$ \\
\hline - Other & $29(30.9)$ \\
\hline Comorbidities: & $78(83)$ \\
\hline - Osteoporosis/osteopenia & $48(51.1)$ \\
\hline - Diabetes & $23(24.5)$ \\
\hline - Mild obesity (BMI $\geq 30)$ & $20(21.3)$ \\
\hline - Arterial hypertension & $20(21.3)$ \\
\hline - Peptic disease & $11(11.7)$ \\
\hline - Chronic coronary heart disease & $9(9.6)$ \\
\hline - Dyslipidaemia & $8(8.5)$ \\
\hline - Psychiatric disorder & $8(8.5)$ \\
\hline - Endocrinopathy & $7(7.4)$ \\
\hline - Non-viral hepatopathy & $7(7.4)$ \\
\hline - Peripheral vasculopathy & $7(7.4)$ \\
\hline - Rheumatic autoimmune disease & $4(4.3)$ \\
\hline - Nephropathy & $3(3.2)$ \\
\hline - Other & $32(34)$ \\
\hline Hospitalization 90 days before LT & $20 / 90(22.2)$ \\
\hline Antibiotic treatment 90 days before LT & $17 / 86(19.8)$ \\
\hline ICU admission 90 days before $\mathrm{LT}$ & $5 / 86(4.7)$ \\
\hline \multicolumn{2}{|l|}{ Type of planned LT } \\
\hline - Single & $52(53.4)$ \\
\hline - Double & $42(44.6)$ \\
\hline \multicolumn{2}{|c|}{ Baseline respiratory bacterial colonization (recipient) } \\
\hline - Overall & $13(13.8)$ \\
\hline - MDR bacteria & $2 / 90(2.2)$ \\
\hline \multicolumn{2}{|c|}{ Baseline respiratory bacterial infection (recipient) } \\
\hline - Overall & $6 / 90(6.6)$ \\
\hline - MDR bacteria & $1 / 90(1.1)$ \\
\hline \multicolumn{2}{|c|}{ Baseline respiratory bacterial colonization (donor) } \\
\hline - Overall & 23/84 (27.4) \\
\hline - MDR bacteria & $2 / 80(2.5)$ \\
\hline \multicolumn{2}{|c|}{ Baseline respiratory bacterial infection (donor) } \\
\hline - Overall & $6 / 81(7.4)$ \\
\hline - MDR bacteria & $0 / 77(0)$ \\
\hline
\end{tabular}

$B M I$, body mass index; $I P F$, idiopathic pulmonary fibrosis; $C O P D$, chronic obstructive pulmonary disease; $L T$, lung transplant; $I C U$, intensive care unit; $M D R$, multi-drug resistant

On the basis of available data, 22.2\% $(n=20 / 90)$ of the recipients had been hospitalized within 90 days before surgery, of whom $17.1 \%(n=15 / 88)$ in the previous 30 days. A limited percentage of the population $(n=5 / 86,5.8 \%)$ had been admitted to intensive care units (ICU) during pre-LT hospitalization. Antibiotic therapy had been prescribed in a limited number of patients within 90 days preceding the transplant ( $n=17 / 86,19.8 \%)$, mostly in the week before the transplant $(n=12 / 79,15.2 \%)$.

Post-transplant follow-up After surgery, ventilation was administered initially by endotracheal tube in all cases. The mean time of intubation was $96 \mathrm{~h}$ (IQR 48-168 h). In $23.3 \%$ ( $n=20 / 86$ ) of patients, a tracheostomy was placed during the hospitalization. Twenty-four of 85 patients (38.2\%) needed surgical revision, after a median time of 11.5 days (IQR 6.526.3) from transplant.

Acute rejection was observed in 26 cases (27.7\%), after a median time of 23.5 days from the surgery (IQR $17-$ 36.3 days). On the total of available histologic referrals, $34.6 \%$ of patients $(n=9)$ were classified as grade $1,38.4 \%$ $(n=10)$ as grade $2,3.8 \%$ as grade $3(n=1), 3.8 \%$ as grade 4 $(n=1)$. A percentage of $7.6(n=2 / 26)$ had a humoral rejection.

PGD occurred in $69 \%$ of cases $(n=64)$, mostly grade 2 $(32.3 \%)$. During the study period $83 \%(n=78)$ underwent a standard systemic antibiotic prophylaxis regimen, while the remaining $17 \%(n=16)$ received a different scheme of prophylaxis based on previous microbiological isolates and/or allergies. Mean duration over the study period was 15 days (IQR 11-18) for anti-Gram negative agents and 12 days (IQR 8-18) for anti-Gram positive drugs. Overall, 87.2\% $(n=82)$ received aerosol prophylaxis after the transplant, for a mean duration of 29 days (IQR 20-43 days). Of this group, 89\% ( $n=73 / 82)$ received taurolidine as aerosol prophylaxis, for a mean time of 30 days (IQR 21.5-45 days).

\section{Bacterial respiratory colonization after transplantation} Thirty-three percent of the population $(n=31)$ developed bacterial colonization of the lower respiratory airways within the first month from LT. On the total of patients with available antimicrobial susceptibility test of post-LT isolates, $11.4 \%$ ( $n=10 / 88$ ) were colonized by MDR bacteria and one by an XDR bacterium. No PDR isolates were found. The most frequent MDR isolates after the transplant were Stenotrophomonas maltophilia $(n=4)$, MRSA $(n=3)$, Enterococcus fecalis $(n=2)$. One XDR Klebsiella pneumoniae was isolated, susceptible only to gentamicin and colistin.

A significant increase in overall and MDR/XDR bacterial colonization was revealed comparing pre- and post-LT period: respectively, $13.8 \%(n=13 / 94)$ versus $33 \%(n=31 / 94)$, $p<0.0001$ and $2.2 \%(n=2 / 88)$ versus $12.5 \%(n=11 / 88)$, $p<0.0001$.

Differences between patients showing bacterial respiratory colonization (all bacteria and MDR bacteria) during the first month after transplantation are showed in Table 2. Patients 
Table 2 Risk factors for bacterial respiratory colonization 30 days after LT

\begin{tabular}{|c|c|c|c|c|c|c|}
\hline Risk factors & $\begin{array}{l}\text { 30-day } \\
\text { colonization } \\
N=31(\%)\end{array}$ & $\begin{array}{l}\text { No } 30 \text {-day coloniza- } \\
\text { tion } \\
N=63(\%)\end{array}$ & $\begin{array}{l}p \\
\text { value }\end{array}$ & $\begin{array}{l}\text { 30-day MDR } \\
\text { colonization } \\
N=10(\%)\end{array}$ & $\begin{array}{l}\text { No 30-day MDR coloniza- } \\
\text { tion } \\
N=78(\%)\end{array}$ & $\begin{array}{l}p \\
\text { value }\end{array}$ \\
\hline Males & $21(67.7)$ & $38(60.3)$ & 0.48 & $7(70)$ & $29(37.2)$ & 0.74 \\
\hline Median age (IQR) & $55(49.61)$ & $58(52-62)$ & 0.16 & $59(54.8-64.3)$ & $48.5(47.8-52)$ & 0.29 \\
\hline Pre-LT BMI & $24(20-30)$ & $24(20-28)$ & 0.65 & $25.4(20-30.5)$ & $24(20-27.3)$ & 0.30 \\
\hline \multicolumn{2}{|l|}{ Indication for transplant: } & & 0.72 & & & 0.07 \\
\hline - IPF & $15(48.4)$ & $26(41.3)$ & & $8(80)$ & $32(41)$ & \\
\hline - COPD & $8(25.8)$ & $16(25.4)$ & & $1(10)$ & $20(25.6)$ & \\
\hline - Other & $8(25.8)$ & $21(33.3)$ & & $1(10)$ & $26(33.3)$ & \\
\hline \multicolumn{2}{|l|}{ Type of transplant: } & & 0.02 & & & 0.51 \\
\hline - Single & $12(38.7)$ & $40(63.5)$ & & $7(70)$ & $43(55.1)$ & \\
\hline - Double & $19(61.3)$ & $23(36.5)$ & & $3(30)$ & $35(44.9)$ & \\
\hline Comorbidities: & $26(83.9)$ & $52(82.5)$ & 0.87 & $8(80)$ & $65(83.3)$ & 0.68 \\
\hline - Osteoporosis/osteopenia & $18(58.1)$ & $30(47.6)$ & 0.34 & $5(50)$ & $38(48.7)$ & 0.94 \\
\hline - Peptic disease & $6(19.4)$ & $5(7.9)$ & 0.11 & $4(40)$ & $6(7.7)$ & 0.01 \\
\hline - Chronic coronary heart disease & $6(19.4)$ & $3(4.8)$ & 0.06 & $2(20)$ & $7(9)$ & 0.27 \\
\hline - Diabetes & $7(22.6)$ & $16(25.4)$ & 0.77 & $3(30)$ & $19(24.4)$ & 0.71 \\
\hline - Obesity & $8(25.8)$ & $12(19)$ & 0.45 & $4(40)$ & $14(17.9)$ & 0.20 \\
\hline - Nephropathy & $1(3.2)$ & $2(3.2)$ & 0.42 & $0(0)$ & $2(2.6)$ & 1 \\
\hline Hospitalization 90 days before LT & $6(19.4)$ & $14 / 59(23.7)$ & 0.32 & $2 / 10(20)$ & $17 / 74(23)$ & 0.68 \\
\hline ICU admission 90 days before LT & $6(19.4)$ & $3 / 57(5.3)$ & 0.82 & $0 / 9(0)$ & $4 / 72(5.5)$ & 0.75 \\
\hline $\begin{array}{l}\text { Antibiotic treatment } 90 \text { days before } \\
\text { LT }\end{array}$ & $3 / 30(10)$ & $14 / 56(25)$ & 0.11 & $1(10)$ & $16 / 71(22.5)$ & 0.39 \\
\hline \multicolumn{7}{|c|}{ Baseline respiratory bacterial colonization (recipient): } \\
\hline - Overall & $4(12.9)$ & $9(14.3)$ & 0.81 & $2(20)$ & $10(12.8)$ & 0.62 \\
\hline - MDR bacteria & $0 / 29(0)$ & 2/61 (3.3) & 0.47 & $0 / 9(0)$ & $2 / 76(2.6)$ & 0.14 \\
\hline \multicolumn{7}{|c|}{ Baseline respiratory bacterial infection (recipient): } \\
\hline - Overall & $2(6.5)$ & $4 / 59(6.7)$ & 0.34 & $1(10)$ & $5 / 74(6.8)$ & 0.54 \\
\hline - MDR bacteria & $0(0)$ & $1 / 59(1.7)$ & 0.63 & $0 / 9(0)$ & $1 / 74(1.4)$ & 0.77 \\
\hline \multicolumn{7}{|c|}{ Baseline respiratory bacterial colonization (donor): } \\
\hline - Overall & $10 / 28(35.7)$ & $13 / 56(23.3)$ & 0.24 & 2/8 (25) & $18 / 70(25.7)$ & 1 \\
\hline - MDR bacteria & $1 / 26(3.8)$ & $1 / 50(1.9)$ & 0.84 & $0 / 8(0)$ & $2 / 67(3)$ & 1 \\
\hline \multicolumn{7}{|c|}{ Baseline respiratory bacterial infection (donor): } \\
\hline - Overall & $0 / 27(0)$ & $6 / 54(11.1)$ & 0.19 & $0 / 8(0)$ & $6 / 67(9)$ & 1 \\
\hline - MDR bacteria & $0 / 27(0)$ & $0 / 50(0)$ & 0.36 & $0 / 8(0)$ & $0 / 63(0)$ & 1 \\
\hline \multicolumn{7}{|c|}{ Post-LT systemic antibiotic prophylaxis } \\
\hline \multirow[t]{2}{*}{-Atypical } & $23(74.2)$ & $55(87.3)$ & 0.11 & $7(70)$ & $67(85.9)$ & 0.194 \\
\hline & $8(25.8)$ & $8(12.7)$ & & $3(30)$ & $11(14.1)$ & \\
\hline Aerosol prophylaxis & $27(87.1)$ & $55(87.3)$ & 0.98 & $10(100)$ & $66(84.6)$ & 0.35 \\
\hline length of days median (IQR) & $25(17-32)$ & $29(21-44)$ & 0.14 & $17(6.5-31.5)$ & $29(22-44.5)$ & 0.01 \\
\hline Basiliximab induction & $14(45.2)$ & $20(31.7)$ & 0.20 & $3(30)$ & $7(34.6)$ & 1 \\
\hline \multicolumn{7}{|l|}{ Immunosuppressive regimen: } \\
\hline - Tacrolimus & $11 / 28(39.3)$ & $11 / 55(39.3)$ & 0.53 & 3/9 (33.3) & $41 / 68(60.3)$ & 0.84 \\
\hline - Cyclosporine & $17 / 28(60.7)$ & $17 / 55(60.7)$ & & $6 / 9(66.7)$ & $25 / 68(36.8)$ & \\
\hline - Other & $0 / 28(0)$ & $2 / 55(3.6)$ & & $0 / 9(0)$ & $2 / 68(2.9)$ & \\
\hline Rejection & $9(29)$ & $17(27)$ & 0.84 & $3(30)$ & $20(25.6)$ & 0.72 \\
\hline Days from LT, median (IQR) & $24(18.5-38.5)$ & $22(17-37)$ & 0.69 & $43(20-50)$ & $22.5(16.5-34.8)$ & 0.30 \\
\hline PGD & $21(67.7)$ & $43(69.4)$ & 0.87 & $7(70)$ & $51 / 77(66.2)$ & 1 \\
\hline Lenght of stay, days median (IQR) & $36(28-60)$ & $37(29-66)$ & 0.76 & $36.5(28-61.3)$ & $55.5(27.6-75.5)$ & 0.40 \\
\hline
\end{tabular}

$B M I$, body mass index; $I P F$, idiopathic pulmonary fibrosis; $C O P D$, chronic obstructive pulmonary disease; $L T$, lung transplant; $M D R$, multi-drug

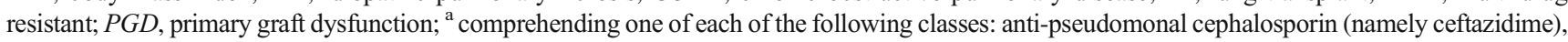
anti-Gram positive drug (namely vancomycin), antifungal prohylaxis (voriconazole), and trimethoprim-sulfamethoxazole prophylaxis

with overall bacterial respiratory colonization had most probably received bilateral LT ( $61.3 \%$ vs. $36.5 \%$ of those not colonized, $p=0.02$ ). On the other side, subjects colonized by MDR had more frequently peptic diseases $(40 \%$ vs. $7.7 \%$ in those not colonized by MDR, $p=0.01$ ) and a shorter length of aerosol prophylaxis (29 days vs. 17 days, $p=0.01$ ).
Factors associated to bacterial colonization (both overall and by MDR strains) during the first month post-LT were investigated by logistic regression; results are summarized in Table 3. Bilateral LT [adjusted odd ratio (AOR) 3.61, 95\% confidence intervals (CI) $1.35-9.60, p=0.01]$ and chronic coronary heart disease (AOR 6.67, 95\% CI 1.36-32.82 $p=$ 
0.02) were independently associated to a higher risk of overall bacterial colonization. On the other side, peptic diseases (AOR 7.66, 95\% CI 1.48-39.77, $p=0.01$ ) was associated with a higher risk of colonization by MDR strains, while those with longer duration of aerosol prophylaxis showed a lower risk of MDR colonization (AOR 0.95 per 1 day increase, $95 \%$ CI $0.90-1.00, p=0.04)$.

A further analysis comparing patients not colonized versus those colonized by susceptible strains versus those colonized by MDR/XDR bacteria is provided as supplementary material, confirming peptic diseases as a risk factor for 30 days MDR colonization (Supplementary Table 1).

Bacterial respiratory infections after transplantation Overall, $35.5 \%$ of lung recipients $(n=33)$ developed bacterial pneumonia, and in more than half of the cases $(n=19 / 33 ; 57.6 \%)$ MDR bacteria were the responsible agents. The main MDR strains isolated in case of pneumonia were MRSA $(n=6)$, Pseudomonas aeruginosa $(n=5)$, Enterococcus faecium $(n=2)$, E. coli $(n=2)$, Acinetobacter baumannii $(n=1)$. No XDR pathogen was isolated during infections in the post-LT observed period.

Differences between patients showing bacterial respiratory infection (all bacteria and MDR bacteria) during the first month after transplantation are showed in Table 4. Patients developing pneumonia in the early posttransplant period had more frequently a history of previous infection in the pre-transplant period $(21.2 \%$ vs. $0 \%, p=$ 0.01 ) and a longer length of hospital stay (median 51 vs. 34 days, $p=0.03$ ). Pre-LT diagnosis of IPF was a significant risk factor for developing both $\operatorname{MDR}(p<0.01)$ and non-MDR infections $(p=0.03)$. In MDR infected population a higher prevalence of PGD (all grades) was reported: $94.7 \%$ of recipients $(n=18 / 19)$ vs. $63.4 \%(n=45 / 72)$ of those not MDR infected, $p=0.01$.

Factors associated to bacterial infection (both overall and by MDR strains) during the first month post-LT were investigated by logistic regression; results are summarized in
Table 5. COPD (AOR 0.17 versus IPF, 95\% CI 0.05-0.66, $p=0.01$ ) and higher BMI (AOR 0.87 per $1 \mathrm{~kg} / \mathrm{m}^{2}$ increase, $95 \%$ CI $0.78-0.98, p=0.03$ ) were associated to a lower risk of bacterial infection, while aerosol prophylaxis with taurolidine was associated to a higher risk (AOR 5.86 versus no aerosol prophylaxis, 95\% CI 1.05-32.69, $p=0.04)$. Narrowing the analysis to MDR infected patients, patients with IPF confirmed a higher risk of infection ( $p<0.05$, see Table 5); a higher risk of MDR infection was also observed in those with pre-transplant colonization (AOR 5.37, 95\% CI 1.02-28.28, $p=0.04$ ) and pre-transplant infections (AOR 16.84, 95\% CI 1.18-239.39, $p=0.01)$.

Both baseline bacterial colonization and baseline respiratory infections were statistically related to MDR respiratory infections within 30 days after LT (see Table 5). Specifically, in $1 / 2(50 \%)$ pre-LT MDR colonized patients and in $1 / 11(9.1 \%)$ pre-LT non-MDR colonized patients, the same bacteria were responsible of post-LT infections, respectively MDR S. aureus and non-MDR S. marcescens. Moreover, 2/5 (40\%) patients with pre-LT non-MDR infection developed post-LT infection by the same bacteria ( 1 E. coli and 1 $P$. aeruginosa). The only one patient with pre-LT pneumonia sustained by MDR P. aeruginosa was colonized by the same bacterium in the post-LT BAL specimens but did not develop an infection.

On the total of 31 post-LT colonized patients, 5/10 (50\%) MDR colonized and 6/21 (28.6\%) non-MDR colonized patients developed infections: in 2 cases, infection was caused by the same colonizing bacteria (respectively 1 MDR S. aureus and 1 non-MDR E. faecalis).

\section{Immunosuppressive regimens and microbiological outcomes} All patients received corticosteroid therapy consisting of intravenous methylprednisolone $125 \mathrm{mg}$ before graft reperfusion followed by $375 \mathrm{mg}$ on day 0 and tapering from $1 \mathrm{mg} / \mathrm{kg}$ on day 1 . Induction therapy was introduced in our protocol in 2009; however, since then not all patients received it, depending on the decision of the surgeon.
Table 3 Risk factors for respiratory bacterial colonization at 30-day post-LT (logistic regression)

\begin{tabular}{|c|c|c|c|c|c|c|}
\hline \multicolumn{3}{|l|}{ Univariate } & \multicolumn{4}{|c|}{ Multivariate } \\
\hline & OR & CI $95 \%$ & $p$ value & $\mathrm{aOR}$ & CI $95 \%$ & $p$ value \\
\hline \multicolumn{7}{|l|}{ Overall 30-day colonization } \\
\hline Double LT & 2.75 & $1.11-6.68$ & 0.02 & 3.61 & $1.35-9.60$ & 0.01 \\
\hline Chronic coronary heart disease & 4.80 & $1.11-20.72$ & 0.04 & 6.67 & $1.36-32.82$ & 0.02 \\
\hline \multicolumn{7}{|l|}{ MDR 30 days colonization } \\
\hline Peptic disease & 8 & $1.76-36.38$ & 0.007 & 7.66 & $1.48-39.77$ & 0.01 \\
\hline $\begin{array}{l}\text { Aerosol prophylaxis, mean } \\
\text { time, per 1-day increase }\end{array}$ & 0.95 & $0.91-1.00$ & 0.046 & 0.95 & $0.90-1.00$ & 0.04 \\
\hline
\end{tabular}

$L T$, lung transplant; $M D R$, multi-drug resistant bacteria 
Table 4 Risk factors for bacterial respiratory infection 30-days after LT

\begin{tabular}{|c|c|c|c|c|c|c|}
\hline Risk factors & $\begin{array}{l}\text { 30-day infection } \\
N=33(\%)\end{array}$ & $\begin{array}{l}\text { Not infected } \\
N=60(\%)\end{array}$ & $p$ value & $\begin{array}{l}\text { 30-day MDR infection } \\
N=19(\%)\end{array}$ & $\begin{array}{l}\text { Not MDR infected } \\
N=72(\%)\end{array}$ & $p$ value \\
\hline Males & $17(51.5)$ & $41(68.3)$ & 0.11 & 11(57.9) & $46(63.9)$ & 0.63 \\
\hline Median age (IQR) & $57(48.5-61.5)$ & $56(51-61)$ & 0.70 & $60(55-63)$ & $56(49-60.8)$ & 0.10 \\
\hline Pre-transplant BMI & $23(20-25.7)$ & $24.4(20.1-30)$ & 0.53 & $24(20-27)$ & $24(20-29)$ & 0.98 \\
\hline Indication for transplant: & & & 0.03 & & & $<0.01$ \\
\hline $\begin{array}{l}\text { - IPF } \\
\text { - COPD } \\
\text { - Other }\end{array}$ & $\begin{array}{l}20(60.6) \\
4(12.2) \\
9(27.3)\end{array}$ & $\begin{array}{l}21(35) \\
19(31.7) \\
20(33.3)\end{array}$ & & $\begin{array}{l}14(73.7) \\
1(5.3) \\
4(21.1)\end{array}$ & $\begin{array}{l}26(36.1) \\
22(30.6) \\
24(33.3)\end{array}$ & \\
\hline Type of transplant: & & & 0.81 & & & 0.26 \\
\hline $\begin{array}{l}\text { - Single } \\
\text { - Double }\end{array}$ & $\begin{array}{l}19(57.6) \\
14(42.4)\end{array}$ & $\begin{array}{l}33(55) \\
27(45)\end{array}$ & & $\begin{array}{l}13(68.4) \\
6(31.6)\end{array}$ & $\begin{array}{l}39(54.2) \\
33(45.8)\end{array}$ & \\
\hline $\begin{array}{l}\text { Comorbidity: } \\
\text { - Osteoporosis/osteopenia }\end{array}$ & $\begin{array}{l}25(75.8) \\
14(42.4)\end{array}$ & $\begin{array}{l}52(86.7) \\
33(55)\end{array}$ & $\begin{array}{l}0.18 \\
0.25\end{array}$ & $\begin{array}{l}15(78.9) \\
8(42.1)\end{array}$ & $\begin{array}{l}61(84.7) \\
38(52.8)\end{array}$ & $\begin{array}{l}0.51 \\
0.41\end{array}$ \\
\hline - Peptic disease & $5(15.2)$ & $6(10)$ & 0.46 & $4(21.1)$ & $7(9.7)$ & 0.23 \\
\hline - Chronic coronary heart disease & $3(9.1)$ & $6(10)$ & 1 & $2(10.5)$ & $7(9.7)$ & 1 \\
\hline - Diabetes & $6(18.2)$ & $17(28.3)$ & 0.28 & $5(26.3)$ & $18(25)$ & 0.91 \\
\hline - Obesity & $4(12.1)$ & $16(26.7)$ & 0.12 & $4(21.1)$ & $16(22.2)$ & 1 \\
\hline - Nephropathy & $2(6.1)$ & $1(1.7)$ & 0.25 & $1(5.3)$ & $2(2.8)$ & 0.51 \\
\hline Hospitalization 90 days before $\mathrm{LT}$ & $9(27.3)$ & $11(19.6)$ & 0.41 & $5(26.3)$ & $14(20.6)$ & 0.59 \\
\hline ICU admission days before LT & $1(3)$ & $3 / 52(5.8)$ & 1 & $0(0)$ & $4 / 64(6.3)$ & 0.16 \\
\hline Antibiotic treatment 90 days before $\mathrm{LT}$ & $5 / 31(16.1)$ & $12 / 54(22.2)$ & 0.64 & $3 / 17(17.6)$ & $13 / 66(19.7)$ & 0.94 \\
\hline \multicolumn{7}{|c|}{ Baseline respiratory bacterial colonization (recipient): } \\
\hline - Overall & $7(21.2)$ & $7(11.7)$ & 0.22 & $6(31.6)$ & $8(11.1)$ & 0.03 \\
\hline - MDR bacteria & $2 / 31(6.5)$ & $0 / 58(0)$ & 0.12 & $2 / 18(11.1)$ & $0 / 69(0)$ & 0.04 \\
\hline \multicolumn{7}{|c|}{ Baseline respiratory bacterial infection (recipient): } \\
\hline - Overall & $7(21.2)$ & $0 / 56(0)$ & $<0.01$ & $4 / 19(21.1)$ & $2 / 68(2.9)$ & 0.02 \\
\hline - MDR & $1 / 32(3.1)$ & $0 / 56(0)$ & 0.31 & $1 / 18(5.6)$ & $0 / 68(0)$ & 0.15 \\
\hline \multicolumn{7}{|c|}{ Baseline respiratory bacterial colonization (donor): } \\
\hline - Overall & $8 / 32(25)$ & $15 / 51(29.4)$ & 0.66 & $6(31.6)$ & $17 / 62(27.4)$ & 0.73 \\
\hline - MDR bacteria & $1 / 32(3.1)$ & $1 / 47(2.1)$ & 1 & $1(5.3)$ & $1 / 58(1.7)$ & 0.44 \\
\hline \multicolumn{7}{|c|}{ Baseline respiratory bacterial infection (donor): } \\
\hline - Overall & $3 / 31(9.7)$ & $3 / 49(6.1)$ & 0.67 & 2/18 (11.1) & $4 / 60(6.7)$ & 0.62 \\
\hline - MDR bacteria & $0 / 29(0)$ & $0 / 47(0)$ & 0.40 & $3(15.8)$ & $14(23.3)$ & 1 \\
\hline \multicolumn{7}{|l|}{ Systemic antibiotic prophylaxis: } \\
\hline $\begin{array}{l}\text { - Standard } \\
\text { - Atypical } \\
\text { Aerosol prophylaxis } \\
\text { length of days median (IQR) }\end{array}$ & $\begin{array}{l}24(72.7) \\
9(27.3) \\
32(97) \\
32(21.3-46.8)\end{array}$ & $\begin{array}{l}53(88.3) \\
7(11.7) \\
49(81.7) \\
25(18-40.5)\end{array}$ & $\begin{array}{l}0.05 \\
0.15\end{array}$ & $\begin{array}{l}13(68.4) \\
6(31.6) \\
19(100) \\
33(22-51)\end{array}$ & $\begin{array}{l}62(86.1) \\
10(13.9) \\
60(83.8) \\
28.5(18.5-41.8)\end{array}$ & $\begin{array}{l}0.07 \\
0.19\end{array}$ \\
\hline Basiliximab induction & $13(39.4)$ & $20(33.3)$ & 0.56 & $6(31.6)$ & $26 / 72(36.1)$ & 0.71 \\
\hline \multicolumn{7}{|l|}{ Immunosuppressive regimen: } \\
\hline $\begin{array}{l}\text { - Tacrolimus } \\
\text { - Cyclosporine } \\
\text { - Other } \\
\text { Rejection }\end{array}$ & $\begin{array}{l}10 / 29(34.5) \\
19 / 29(65.5) \\
0 / 29(0) \\
10(30.3)\end{array}$ & $\begin{array}{l}18 / 53(34) \\
33 / 53(62.3) \\
2 / 53(3.8) \\
16(26.7)\end{array}$ & 0.57 & $\begin{array}{l}6 / 16(37.5) \\
10 / 16(62.5) \\
0 / 16(0) \\
7(36.8)\end{array}$ & $\begin{array}{l}21 / 64(32.8) \\
41 / 64(64.1) \\
2 / 64(3.1) \\
19(26.4)\end{array}$ & 0.74 \\
\hline Days from LT, median (IQR) & $22(12.8-38.5)$ & $24(19.3-34.8)$ & 0.55 & $23(17-43)$ & $24(16-35.5)$ & 0.80 \\
\hline PDG & $27(81.8)$ & $37(62.7)$ & 0.06 & $18(94.7)$ & $45(63.4)$ & 0.01 \\
\hline Length of stay, days median (IQR) & $51(32-74)$ & $34.5(27-54.8)$ & 0.03 & $51(33-70)$ & $35.5(28-56.5)$ & 0.15 \\
\hline
\end{tabular}

$B M I$, body mass index; $I P F$, idiopathic pulmonary fibrosis; $C O P D$, chronic obstructive pulmonary disease; $L T$, lung transplant; $M D R$, multi-drug resistant; $P G D$, primary graft dysfunction; ${ }^{\text {a }}$ comprehending one of each of the following classes: anti-pseudomonal cephalosporin (namely ceftazidime), anti-Gram positive drug (namely vancomycin), antifungal prophylaxis (voriconazole), and trimethoprim-sulfamethoxazole prophylaxis 
Table 5 Risk factors for respiratory bacterial infection at 30-day post-LT (logistic regression)

\begin{tabular}{|c|c|c|c|c|c|c|}
\hline & \multicolumn{4}{|c|}{ Univariate } & \multicolumn{2}{|l|}{ Multivariate } \\
\hline & OR & $95 \% \mathrm{CI}$ & $p$ value & $\mathrm{aOR}$ & $95 \% \mathrm{CI}$ & $p$ value \\
\hline \multicolumn{7}{|l|}{ 30-day overall infection } \\
\hline \multicolumn{7}{|l|}{ Transplant indication } \\
\hline - COPD vs. IPF & 0.22 & $0.06-0.76$ & 0.02 & 0.17 & $0.05-0.66$ & 0.01 \\
\hline - Others vs. IPF & 0.47 & $0.17-1.28$ & 0.14 & 0.52 & $0.17-1.59$ & 0.25 \\
\hline BMI (per $1 \mathrm{~kg} / \mathrm{m}^{2}$ increase) & 0.90 & $0.81-1.00$ & 0.049 & 0.87 & $0.78-0.98$ & 0.03 \\
\hline \multicolumn{7}{|l|}{ Aerosol } \\
\hline - No aerosol prophylaxis & REF & REF & REF & REF & REF & REF \\
\hline - Taurolidine aerosol & 4.5 & $0.95-21.38$ & 0.06 & 5.86 & $1.05-32.69$ & 0.04 \\
\hline - Taurolidine + other antibiotic & 3.5 & $0.21-51.77$ & 0.38 & 5.72 & $0.29-110.7$ & 0.24 \\
\hline $\begin{array}{l}\text { Antibiotic aerosol in addition to } \\
\text { systemic therapy }\end{array}$ & 10.5 & $1.03-107.17$ & 0.047 & 10.32 & $0.81-130.33$ & 0.07 \\
\hline Atypical $^{\mathrm{a}}$ prophylaxis & 2.83 & $0.94-8.52$ & 0.06 & 2.27 & $0.64-8.07$ & 0.20 \\
\hline \multicolumn{7}{|l|}{ 30-days MDR infection } \\
\hline \multicolumn{7}{|l|}{ Transplant indication: } \\
\hline - COPD vs. IPF & 0.08 & $0.01-0.84$ & 0.02 & 0.07 & $0.01-0.77$ & 0.03 \\
\hline - Other vs. IPF & 0.31 & $0.08-1.10$ & 0.06 & 0.17 & $0.03-0.86$ & 0.03 \\
\hline Pre-LT respiratory colonization & 3.69 & $0.78-16.88$ & 0.03 & 5.37 & $1.02-28.28$ & 0.04 \\
\hline Pre-LT respiratory infection & 8.8 & $0.59-1.40$ & 0.02 & 16.84 & $1.18-239.39$ & 0.04 \\
\hline PGD & 10.4 & $1.13-82.48$ & 0.03 & 6.45 & $0.75-56.37$ & 0.09 \\
\hline Atypical ${ }^{\mathrm{a}}$ prophylaxis & 2.86 & $0.88-9.27$ & 0.08 & 1.83 & $0.34-9.92$ & 0.48 \\
\hline
\end{tabular}

$C O D P$, chronic obstructive pulmonary disease; $I P F$, interstitial pulmonary fibrosis; $B M I$, body mass index; $P G D$, primary graft dysfunction; $R E F$, reference for risk factors analysis; $M D R$, multi-drug resistant; ${ }^{a}$ not comprehending at least one of the following: anti-pseudomonal cephalosporins (mainly ceftazidime), antiGram positive drug (mainly vancomycin), antifungal (mainly voriconazole), or trimethoprim-sulfamethxazole prohylaxis
One-third of patients $(36.2 \% n=34 / 94)$ were treated with basiliximab (20 $\mathrm{mg}$ on day 0 and day 4$)$; only a few $(2.6 \%$, $n=2 / 94)$ received rabbit antithymocyte globulin $(1.5 \mathrm{mg} / \mathrm{kg} /$ day for 3-5 days).

Calcineurin inhibitors were introduced on days 3-5 using either tacrolimus (trough level 10-15 $\mathrm{ng} / \mathrm{ml}$ before introduction of basiliximab, $8-10 \mathrm{ng} / \mathrm{ml}$ thereafter) or cyclosporine (trough level 250-300 ng/ml before introduction of basiliximab, 200-250 $\mathrm{ng} / \mathrm{ml}$ thereafter). Cyclosporine was used predominantly until 2007; tacrolimus was used thereafter. Azathioprine $100 \mathrm{mg} /$ day or mycophenolate mofetil $1 \mathrm{~g}$ /day was introduced in most patients from day 7 to day 10 . In 2007, mycophenolate mofetil replaced azathioprine in the baseline regimen for all patients.

Rates of colonization or infection (both globally and by MDR) at 30 days post-LT were not significantly different when comparing subgroups treated with different immunosuppressive regimens (cyclosporine versus tacrolimus versus all other regimen) or subgroups with or without basiliximab induction (see Tables 2 and 4).
Antibiotic prophylaxis schemes and microbiological outcomes Patients treated with standard systemic antibiotic prophylaxis did not show significantly different 30 days post-LT bacterial colonization rates when compared to those receiving alternative regimens [overall colonization: $29.5 \%$ (23/78) versus $50 \%(8 / 16), p=0.112$; MDR colonization: $9.5 \%(7 / 74)$ versus $21.4 \%(3 / 14), p=0.194]$.

Higher rates of 30-days post-LT bacterial infection were reported in the subgroup of patients receiving an alternative prophylaxis scheme when compared to those treated with standard prophylaxis [overall infections: $56.3 \%$ (9/16) versus $31.2 \%(24 / 77), p=0.056]$. Infections by MDR bacteria were diagnosed in $17.3 \%(13 / 75)$ of patients receiving standard prophylaxis regimen versus $37.5 \%(6 / 16)$ of patients receiving an alternative scheme $(p=0.072)$. However, these trends toward an association were not confirmed in multivariate analyses (see Table 5).

No significant differences regarding colonization and infection rates (overall and by MDR) were observed between patients receiving aerosolized antibiotics versus those not receiving them (data not shown). 


\section{Discussion}

Graft colonization and infection in lung transplant recipients represent a common finding [13], with a higher risk observed especially in the first months after surgery [8]. In early postsurgery, the lung allograft is particularly susceptible to infections: the epithelium dysfunction related to the disruption of bronchial circulation and lymphatic drainage, the denervation of the allograft causing a loss of cough reflex and bronchial hyperresponsivity, and the possible stenosis or necrosis of the bronchial anastomosis may facilitate colonization or infection of airways mucosae and/or lung tissue [7]. Infections are the most common cause of death in lung recipients [1]. Most of the related literature on post-transplant infection management is based on experts' opinion and single center retrospective studies, especially in Europe, but a comprehensive global analysis is difficult to achieve. Every day clinical management in different lung transplant centers may differs due to epidemiological peculiarity [21] and local evidence based medicine. Results from each single center could enrich data analysis and offer confrontation for clinical management.

With the limitations of a retrospective specific cohort analysis, our study revealed a significant increase of respiratory bacterial colonization by both overall bacteria $(p<0.0001)$ and by MDR/XDR bacteria $(p<0.0001)$ in the 30 days postLT. A significantly greater percentage of 30-days respiratory infections occurred in patients with a baseline bacterial colonization $(p=0.03)$ or infection $(p=0.02)$.

Donor-derived infections are also of major concern [9]; however, a low risk of donor-recipient MDR transmission has been previously hypothesized [22]. In our cohort, at least $31 \%$ of donors had a respiratory bacterial colonization, with a low percentage of MDR bacteria (2.5\%), but these observations appeared not to be related to colonization and infection in recipients.

In our cohort, the transplant type seemed to play a role in the prediction of post-transplant infectious risk within the first month after LT. In fact, patients with 30 days pulmonary colonization most frequently received baseline double lung transplant $(61.3 \%$ vs. $36.5 \%, p=0.02)$. Discordant data are reported in literature regarding correlation between type of LT, infectious complications, and outcome. Meyer et al. observed that single lung transplant in patients with IPF had a better 30-day and 1-year outcome, although the reasons were not clarified, and a role of early events (e.g., infections and colonizations together with surgical technical procedure) was hypothesized [23]. Other studies suggested that bilateral LT did not differ from single LT in terms of overall mortality, even if single LT could have better rates of long-term survival after transplant in $>60$-year-old recipients $[24,25]$ and, on the other hand, that bilateral LT can offer a survival advantage in younger recipients with end-stage emphysema [24, 26]. Our findings can suffer from biases of a retrospective study; however, reported discordant data underline the need of further analysis regarding correlation of type of LT and infectious risk.

We also found that patients with chronic coronary heart disease were more subject to develop lower respiratory tract bacterial colonization in the first month after surgery. Twenty percent of lung recipients had ischemic heart disease as a baseline comorbidity, and these patients had a sixfold increment of bacterial colonization.

Our results showed also that peptic disease (non-ulcerative gastritis; esophagitis; gastric ulcer; and gastroesophageal reflux disease, GERD) was associated to a higher risk of MDR bacteria colonization in the first month after LT. This finding is in line with previous reports [27, 28], which also highlights a high incidence of peptic disease in lung recipients and a more rapid progression to acute and chronic graft rejection after transplant in patients affected by this condition. This can be theoretically explained by various factors, such as continuous asymptomatic aspiration of peptic material which can lead to direct injuries and trigger inflammatory response within the graft, thus facilitating bacterial colonization.

Interestingly, we also found that duration of nebulized therapy was linked to the risk of MDR colonization. In particular, patients who underwent longer periods of aerosolized antibiotic drugs administration were found to be less prone to develop lung MDR colonization within the first 30 days. No benefits were found about the use of aerosolized taurolidine (a topic decontaminant used in many lung transplant centers) as a prophylactic strategy; indeed, aerosolized taurolidine was associated to a higher risk of bacterial infections. This finding should be interpreted with caution due to the small study population and retrospective design of the study, since taurolidine could have been prescribed to patients at higher risk to develop infections: in our center, taurolidine aerosol is indeed commonly used, in combination with systemic antibiotics, as salvage off label therapy in severe respiratory infections after LT. As a consequence, prospective randomized studies are needed to clarify the role of taurolidine as an effective agent used to prevent bacterial respiratory infections. Nebulized administration of antibiotic can be a helpful additional strategy to decontaminate airways after transplant, especially from Gram negative isolates [29], although data from randomized trials are currently not available. This route of administration can be a way to optimize the treatment, reducing the risk of colonization, especially in the first year, with a limited risk of adverse reactions [29].

Even if not confirmed by multivariate analyses, our data revealed a lower 30 days colonization and infection rates (overall and by MDR bacteria) in patients receiving standard systemic antibiotic prophylaxis (extended spectrum cephalosporin plus a glycopeptide) in comparison with atypical regimens, endorsing the clinical relevance to follow guidelines indications [38], in absence of alternative clinical needs. 
We also found a significant link between the occurrence of pneumonia in the early post-transplant period and body weight of the recipients. A higher baseline BMI was associated to a reduced risk of developing pneumonia. Our finding could be explained by the fact that a major reservoir of lean and fat mass could permit to bear major stresses, such as the surgery, shortening the ICU stay, and supporting recovery period. In addition, underweight, as well as obesity, can lead to recovery problems during post-surgery period and can be linked to a partially harmed immunity function. Our findings are in line with previous reports [30]: a major incidence of post-LT infections was observed in underweighted patients, together with a shorter survival. Optimal baseline BMI seems to be associated with lower mortality rates, especially at 90 days and 1 year [31]. Underweighted and obese patients also seem to be at higher risk of poor outcome in the first year [32]. Obesity of grades II and III, on the other hand, is an absolute contraindication to transplant, while a less strict approach is adopted with mild obesity (grade I) in which transplant can be considered as an option [33]. An ideal baseline body weight is an important factor to estimate infectious risk and to assess the predicted outcome of lung recipients [30].

We found an association between baseline IPF and the development of pneumonia by MDR bacteria. Previous studies reported high rates of pulmonary and bloodstream infection in person affected by pulmonary fibrosis [34, 35]. In our cohort, patients with baseline IPF were more prone to develop respiratory infections than patients with COPD. This could be related to a higher pre-LT immunosuppression: before 2014 steroidal and immunosuppressive drugs (e.g., azathioprine) were the only therapy for IPF. Therefore, a greater cumulative exposure to these drugs could have been linked to the high incidence of comorbidities and infections. This observation could also partly explain the higher post-transplant mortality in IPF patients, which some authors highlighted [4].

A higher incidence of infections by bacteria harboring MDR is known after solid organ transplant [13,36]. An active surveillance program during the pre- and post-transplant period including surface swabs and respiratory secretions culture is fundamental to steadily detect colonizing MDR bacteria, thus optimizing post-transplant antibiotic strategies when needed, particularly in patients with PGD. The cause of PGD is likely multifactorial with brain death-related donor lung injury, ischemia-reperfusion injury, infection, and cardiopulmonary bypass representing some of the possible etiologies. Moreover, infections can represent factors that may confound and/or amplify a diagnosis of primary graft dysfunction [15]. In our study, we observed a significantly higher prevalence of PGD (all grades) in MDR infected patients $(94.7 \%$ versus $63.4 \%$ in non-MDR infected patients, $p=$ $0.01)$.

Our analysis of independent risk predictors identified also a role of pre-transplant respiratory colonization and infection in predicting 30 days pneumonia (respectively AOR 5.43, 95\% CI 1.03-28.54, $p=0.04$, AOR 22.42, 95\% CI 2.22-269.20, $p=0.01$ ), highlighting the role of an accurate pre-transplant microbiological monitoring strategy. Either in pre- and posttransplant period an appropriate use of antibiotics (on the basis of antimicrobial susceptibility testing) is crucial, in order to both prevent possible adverse effects and avoid selective pressure on resistant bacteria $[36,37]$.

In conclusion, it is well known that anamnestic, clinical, and microbiological pre-transplant features are key factors for planning the post-transplant strategies. Recent guidelines [38] have focused on surgical site infection, but in LT an increasing infectious risk is obviously present for respiratory tract infections. Identification of high-risk patients before the LT could guide to personalize prophylaxis and therapy, when needed, and to lead to shorter and tailored antibiotic treatment, reducing adverse effects and selection of MDR strains. On the basis of our results, the risk of post-transplant lung infectious complications could be stratified by considering several factors such as the indication for LT, the type of LT, the presence of certain comorbidities, and the microbiologic assessment before LT. Importantly, our results suggest that a wider use of early nebulized therapies could be useful to prevent potentially harmful colonization from MDR strains, thus potentially lowering infectious risk.

Supplementary Information The online version contains supplementary material available at https://doi.org/10.1007/s10096-021-04153-1.

Acknowledgements We thank Roberta Gagliardi and Aniello Sammartino for help during initial data collection and David Redi for some useful suggestions during preliminary analysis. We really wish to thank every health care worker of the Siena lung transplant team: their competent and passionate effort is essential in the continuum of care.

We dedicate this study in memoriam of Prof. Andrea De Luca (19642019), Professor of Infectious Diseases at Department of Medical Biotechnologies, University of Siena and Director of Division of Infectious Diseases at Siena University Hospital. Prof. Andrea De Luca was a vivid member of lung transplant team as Infectious Disease Specialist. He was an enlightened Scientist and a dedicated Clinician. We would like to remember him as an inspiring Mentor.

Addendum During manuscript revision one of our co-author, Dr. Luca Marchetti, sorely and suddenly passed away. He was the Chief of Anesthesia and Cardio-Thoracic-Vascular Intensive Care Unit, and he really always dedicated his competence and humanity to take the best care of transplanted patients and of critical cases post cardiovascular surgery. We dearly remember him and his valuable work.

Funding Open Access funding provided by Università degli Studi di Siena.

Data availability The datasets used and/or analyzed during the current study are available from the corresponding author on reasonable request.

\section{Compliance with ethical standards}

Conflict of interest MF received speakers' honoraria and support for travel to meetings from Bristol-Myers Squibb (BMS), Gilead, Janssen- 
Cilag, Merck Sharp \& Dohme (MSD), ViiV Healtcare, and fees for attending advisory boards from BMS, Gilead and Janssen-Cilag. FM received support for travel to meetings from Angelini, she is the principal investigator in a study sponsored by TLS (Toscana Life Science) and by GSK Vaccine SRL and she is the contact person for a service contract between GSK Vaccine SRL and Department of Medical Biotechnologies, University of Siena, without receiving any personal remuneration. All the other authors have no conflicts to declare.

Ethical approval The study was approved by the local ethics committee (Azienda Ospedaliera Universitaria Senese, protocol OSS REOS $n^{\circ}$ 12908).

Open Access This article is licensed under a Creative Commons Attribution 4.0 International License, which permits use, sharing, adaptation, distribution and reproduction in any medium or format, as long as you give appropriate credit to the original author(s) and the source, provide a link to the Creative Commons licence, and indicate if changes were made. The images or other third party material in this article are included in the article's Creative Commons licence, unless indicated otherwise in a credit line to the material. If material is not included in the article's Creative Commons licence and your intended use is not permitted by statutory regulation or exceeds the permitted use, you will need to obtain permission directly from the copyright holder. To view a copy of this licence, visit http://creativecommons.org/licenses/by/4.0/.

\section{References}

1. Yusen RD, Edwards LB, Dipchand AI, Goldfarb SB, Kucheryavaya AY, Levvey BJ et al (2016) The Registry of the International Society for Heart and Lung Transplantation: thirtythird adult lung and heart-lung transplant report-2016; focus theme: primary diagnostic indications for transplant. J Heart Lung Transplant 35(10):1170-1184. https://doi.org/10.1016/j. healun.2016.09.001

2. Lund LH, Khush KK, Cherikh WS, Goldfarb SB, Kucheryavaya AY, Levvey BJ et al (2017) The Registry of the International Society for Heart and Lung Transplantation: thirty-fourth adult heart transplantation report-2017; focus theme: allograft ischemic time. J Heart Lung Transplant 36(10):1037-1046. https://doi.org/ 10.1016/j.healun.2017.07.019

3. Bennett D, Fossi A, Bargagli E, Refini RM, Pieroni M, Luzzi L et al (2015) Mortality on the waiting list for lung transplantation in patients with idiopathic pulmonary fibrosis: a single-centre experience. Lung 193(5):677-681. https://doi.org/10.1007/s00408-0159767-x

4. Kistler KD, Nalysnyk L, Rotella P, Esser D (2014) Lung transplantation in idiopathic pulmonary fibrosis: a systematic review of the literature. BMC Pulm Med 14(1):139. https://doi.org/10.1186/ 1471-2466-14-139

5. Bennett D, Fossi A, Marchetti L, Lanzarone N, Sisi S, Refini RM et al (2019) Postoperative acute kidney injury in lung transplant recipients. Interact Cardiovasc Thorac Surg 28(6). https://doi.org/ 10.1093/ICVTS/IVY355

6. Clark NM, Weigt SS, Fishbein MC, Kubak B, Belperio JA, Lynch JP (2018) Fungal infections complicating lung transplantation. Semin Respir Crit Care Med 39(2):227-254. https://doi.org/10. 1055/s-0037-1617443

7. Burguete SR, Maselli DJ, Fernandez JF, Levine SM (2013) Lung transplant infection. Respirology 18(1):22-38. https://doi.org/10. 1111/j.1440-1843.2012.02196.x
8. Fishman JA (2017) Infection in organ transplantation. Am J Transplant 17(4):856-879. https://doi.org/10.1111/ajt.14208

9. Grossi PA (2018) Donor-derived infections, lessons learnt from the past, and what is the future going to bring us. Curr Opin Organ Transplant 23(4):417-422. https://doi.org/10.1097/MOT. 0000000000000551

10. Nakajima T, Palchevsky V, Perkins DL, Belperio JA, Finn PW (2011) Lung transplantation: infection, inflammation, and the microbiome. Semin Immunopathol:135-156. https://doi.org/10. 1007/s00281-011-0249-9

11. Lin CM, Zamora MR (2014) Update on bronchiolitis obliterans syndrome in lung transplantation. Curr Transplant Rep 1(4):282289. https://doi.org/10.1007/s40472-014-0030-9

12. Remund KF, Best M, Egan JJ (2009) Infections relevant to lung transplantation. Proc Am Thorac Soc:94-100. https://doi.org/10. 1513/pats.200809-113GO

13. Gagliotti C, Morsillo F, Moro ML, Masiero L, Procaccio F, Vespasiano $\mathrm{F}$ et al (2018) Infections in liver and lung transplant recipients: a national prospective cohort. Eur J Clin Microbiol Infect Dis 37(3):399-407. https://doi.org/10.1007/s10096-0183183-0

14. Lynch JP, Sayah DM, Belperio JA, Weigt SS (2015) Lung transplantation for cystic fibrosis: results, indications, complications, and controversies. Semin Respir Crit Care Med:299-320. https:// doi.org/10.1055/s-0035-1547347

15. Snell GI, Yusen RD, Weill D, Strueber M, Garrity E, Reed A et al (2017) Report of the ISHLT Working Group on Primary Lung Graft Dysfunction, part I: Definition and grading-A 2016 Consensus Group statement of the International Society for Heart and Lung Transplantation. J Heart Lung Transplant:1097-1103. https://doi.org/10.1016/j.healun.2017.07.021

16. Magiorakos AP, Srinivasan A, Carey RB, Carmeli Y, Falagas ME, Giske CG et al (2012) Multidrug-resistant, extensively drugresistant and pandrug-resistant bacteria: an international expert proposal for interim standard definitions for acquired resistance. Clin Microbiol Infect 18(3):268-281. https://doi.org/10.1111/j.14690691.2011.03570.x

17. Zeriouh M, Sabashnikov A, Patil NP, Schmack B, Zych B, Mohite PN et al (2018) Use of taurolidine in lung transplantation for cystic fibrosis and impact on bacterial colonization. Eur J Cardiothorac Surg 53(3):603-609. https://doi.org/10.1093/ejcts/ezx359

18. De Soyza A, Meachery G, Hester KLM, Nicholson A, Parry G, Tocewicz K et al (2010) Lung transplantation for patients with cystic fibrosis and Burkholderia cepacia complex infection: a single-center experience. J Heart Lung Transplant 29(12):13951404. https://doi.org/10.1016/j.healun.2010.06.007

19. Farid S, Mohamed S, Devbhandari M, Kneale M, Richardson M, Soon SY et al (2013) Results of surgery for chronic pulmonary Aspergillosis, optimal antifungal therapy and proposed high risk factors for recurrence - a National Centre's experience. J Cardiothorac Surg 8(1):180. https://doi.org/10.1186/1749-8090-8180

20. Kosmidis C, Denning DW (2015) The clinical spectrum of pulmonary aspergillosis. Thorax 70:270-277. https://doi.org/10.1136/ thoraxjnl-2014-206291

21. Coiffard B, Prud'Homme E, Hraiech S, Cassir N, Le Pavec J, Kessler R et al (2020) Worldwide clinical practices in perioperative antibiotic therapy for lung transplantation. BMC Pulm Med 20(1): 109. https://doi.org/10.1186/s12890-020-1151-9

22. Errico G, Gagliotti C, Monaco M, Masiero L, Gaibani P, Ambretti S et al (2019) Colonization and infection due to carbapenemaseproducing Enterobacteriaceae in liver and lung transplant recipients and donor-derived transmission: a prospective cohort study conducted in Italy. Clin Microbiol Infect 25(2):203-209. https://doi. org/10.1016/j.cmi.2018.05.003 
23. Meyer DM, Edwards LB, Torres F, Jessen ME, Novick RJ (2005) Impact of recipient age and procedure type on survival after lung transplantation for pulmonary fibrosis. Ann Thorac Surg 79(3): 950-957. https://doi.org/10.1016/j.athoracsur.2004.08.076

24. Subramanian MP, Meyers BF (2018) Bilateral versus single lung transplantation: are two lungs better than one? J Thorac Dis:45884601. https://doi.org/10.21037/jtd.2018.06.56

25. Aryal S, Nathan SDB (2018) Single vs. bilateral lung transplantation. Curr Opin Organ Transplant 23(3):316-323. https://doi.org/ 10.1097/MOT.0000000000000527

26. Meyer DM, Bennett LE, Novick RJ, Hosenpud JD (2001) Single vs bilateral, sequential lung transplantation for end-stage emphysema: influence of recipient age on survival and secondary end-points. J Heart Lung Transplant 20(9):935-941. https://doi.org/10.1016/ S1053-2498(01)00295-9

27. Hathorn KE, Chan WW, Lo WK (2017) Role of gastroesophageal reflux disease in lung transplantation. World J Transplant 7(2):103. https://doi.org/10.5500/wjt.v7.i2.103

28. Lo WK, Goldberg HJ, Wee J, Fisichella PM, Chan WW (2016) Both pre-transplant and early post-transplant antireflux surgery prevent development of early allograft injury after lung transplantation. J Gastrointest Surg 20(1):111-118. https://doi.org/10.1007/s11605015-2983-0

29. Quon BS, Goss CH, Ramsey BW (2014) Inhaled antibiotics for lower airway infections. Ann Am Thorac Soc:425-434. https:// doi.org/10.1513/AnnalsATS.201311-395FR

30. Chamogeorgakis T, Mason DP, Murthy SC, Thuita L, Raymond DP, Pettersson GB et al (2013) Impact of nutritional state on lung transplant outcomes. J Heart Lung Transplant 32(7):693-700. https://doi.org/10.1016/j.healun.2013.04.005

31. Fernandez R, Safaeinili N, Kurihara C, Odell DD, Jain M, DeCamp MM et al (2018) Association of body mass index with lung transplantation survival in the United States following implementation of the lung allocation score. J Thorac Cardiovasc Surg 155(4): 1871-1879.e3. https://doi.org/10.1016/j.jtcvs.2017.11.031
32. Upala S, Panichsillapakit T, Wijarnpreecha K, Jaruvongvanich V, Sanguankeo A (2016) Underweight and obesity increase the risk of mortality after lung transplantation: a systematic review and metaanalysis. Transpl Int:285-296. https://doi.org/10.1111/tri.12721

33. Weill D, Benden C, Corris PA, Dark JH, Davis RD, Keshavjee S et al (2015) A consensus document for the selection of lung transplant candidates: 2014 - An update from the Pulmonary Transplantation Council of the International Society for Heart and Lung Transplantation. J Heart Lung Transplant:1-15. https://doi. org/10.1016/j.healun.2014.06.014

34. George TJ, Arnaoutakis GJ, Shah AS (2011) Lung transplant in idiopathic pulmonary fibrosis. Arch Surg:1204-1209. https://doi. org/10.1001/archsurg.2011.239

35. Vicente R, Morales P, Ramos F, Solé A, Mayo M, Villalain C (2006) Perioperative complications of lung transplantation in patients with emphysema and fibrosis: experience from 1992-2002. Transplant Proc 38(8):2560-2562. https://doi.org/10.1016/j. transproceed.2006.08.048

36. Cervera C, van Delden C, Gavaldà J, Welte T, Akova M, Carratalà J (2014) Multidrug-resistant bacteria in solid organ transplant recipients. Clin Microbiol Infect:49-73. https://doi.org/10.1111/14690691.12687

37. Shoham S, Shah PD (2013) Impact of multidrug-resistant organisms on patients considered for lung transplantation. Infect Dis Clin N Am:343-358. https://doi.org/10.1016/j.idc.2013.02.006

38. Abbo LM, Grossi PA (2019) Surgical site infections: guidelines from the American society of transplantation infectious diseases community of practice. Clin Transpl 33(9). https://doi.org/10. $1111 /$ ctr.13589

Publisher's note Springer Nature remains neutral with regard to jurisdictional claims in published maps and institutional affiliations. 